\title{
Diagnostic Value of Facial Nerve Antidromic Evoked Potential in Patients With Bell's Palsy: A Preliminary Study
}

\author{
Ji Hoon Lee, MD, Sun Mi Kim, MD, Hea Eun Yang, MD, Jang Woo Lee, MD, Yoon Ghil Park, MD, PhD
}

Department of Rehabilitation Medicine and Rehabilitation Institute of Neuromuscular Disease, Yonsei University College of Medicine, Seoul, Korea

Objective To assess the practical diagnostic value of facial nerve antidromic evoked potential (FNAEP), we compared it with the diagnostic value of the electroneurography (ENoG) test in Bell's palsy.

Methods In total, 20 patients with unilateral Bell's palsy were recruited. Between the 1st and 17th days after the onset of facial palsy, FNAEP and ENoG tests were conducted. The degeneration ratio and FNAEP latency difference between the affected and unaffected sides were calculated in all subjects.

Results In all patients, FNAEP showed prolonged latencies on the affected side versus the unaffected side. The difference was statistically significant. In contrast, there was no significant difference between sides in the normal control group. In 8 of 20 patients, ENoG revealed a degeneration ratio less than 50\%, but FNAEP show a difference of more than $0.295 \pm 0.599 \mathrm{~ms}$, the average value of normal control group. This shows FNAEP could be a more sensitive test for Bell's palsy diagnosis than ENoG. In particular, in 10 patients tested within 7 days after onset, an abnormal ENoG finding was noted in only four of them, but FNAEP showed a significant latency difference in all patients at this early stage. Thus, FANEP was more sensitive in detecting facial nerve injury than the ENoG test $(\mathrm{p}=0.031)$.

Conclusion FNAEP has some clinical value in the diagnosis of facial nerve degeneration. It is important that FNAEP be considered in patients with facial palsy at an early stage and integrated with other relevant tests.

Keywords Bell palsy, Electroneurography, Facial nerve, Antidromic evoked potential

Received June 10, 2013; Accepted October 17, 2013

Corresponding author: Yoon Ghil Park

Department of Rehabilitation Medicine, Gangnam Severance Hospital, Yonsei University College of Medicine, 211 Eonju-ro, Gangnam-gu, Seoul 135-720, Korea

Tel: +82-2-2019-3493, Fax: +82-2-3463-7585, E-mail: drtlc@yuhs.ac

(c) This is an open-access article distributed under the terms of the Creative Commons Attribution Non-Commercial License (http://creativecommons. org/licenses/by-nc/3.0) which permits unrestricted noncommercial use, distribution, and reproduction in any medium, provided the original work is properly cited.

Copyright $\odot 2014$ by Korean Academy of Rehabilitation Medicine

\section{INTRODUCTION}

Bell's palsy is caused by damage to the 7th cranial nerve, but in most cases it is idiopathic. It is known to occur due to compression and ischemia resulting from inflammation and edema of the facial nerve, caused by viral infection [1]. Bell's palsy may involve acute unilateral facial palsy, sensory impairment, and drooling. These clinical signs and a physical examination are fundamental 
to the diagnosis of Bell's palsy. A diagnosis of Bell's palsy is typically based on symptoms and by ruling out other disorders, such as central nervous system injury, facial tumors, certain cancers, and autoimmune diseases. Developing over several hours or up to 2-3 days, the typical symptoms include asymmetric smile, slurring of words, muscle weakness, and an inability to close an eye. A definite diagnosis needs electrodiagnostic tests to examine nerve damage and its severity, along with a prognostic prediction. In this regard, electrodiagnostic tests, such as needle electromyography (EMG) and electroneurography (ENoG), have been used in the diagnosis since the 1970s. EMG detects nerve damage, identifying whether spontaneous abnormal activities occur during the resting state in facial muscles, such as the frontalis, obicularis oculi, and orbicularis oris muscles, and whether motor unit action potential changes occur [2-10]. In the case of ENoG, nerve damage is confirmed when waveform amplitude is smaller than half of that on the unaffected side [10-12]. However, both tests may be less accurate when they are performed within a week after the onset of Bell's palsy. This is because they can only examine the extratemporal segment of the facial nerves [2-10].

Early treatment is crucial in recovery from Bell's palsy, which needs early detection of nerve damage. A facial nerve antidromic evoked potential (FNAEP) study is the only electrodiagnostic tool for evaluating the intratemporal segment of the facial nerves, so it is effective to diagnose the early stage of Bell's palsy, within a week of onset [13]. However, previous studies examining FNAEP have been conducted in animal experiments [14,15], most of which were focused on FNAEP waveforms [13], and there are very few reported studies on the diagnostic value of FNAEP latency. In this study, we sought to determine a reference value for FNAEP latency and to compare FNAEP and ENoG in terms of diagnostic value in Bell's palsy, especially in the early stages, within 1 week.

\section{MATERIALS AND METHODS}

This study was conducted on 20 patients with Bell's palsy from March to December 2012, who underwent FNAEP and ENoG tests on the same day. Written informed consent was obtained from all subjects prior to the study.

Before the examination, to check the severity of the facial nerve injury, we used the House-Brackmann scale between I (normal) and VI (no movement) [16]. FNAEP was used to measure the latency difference between affected side and unaffected side, and ENoG was to calculate the degeneration ratio of the facial nerve compared with the unaffected side. ENoG was performed using the Medelec Synergy EMG equipment (Oxford Instrument Medical Ltd., Surrey, UK) with a built-in monitor, bipolar stimulator, and discoid electrodes. Facial nerve trunks were stimulated by supramaximal stimuli through the bipolar stimulator at the stylomastoid foramens, and compound muscle action potentials (CMAPs) were measured through recording electrodes placed on the ala nasi. The reference electrode was placed on the nasolabial folds (with a 2-cm interelectrode distance). The ground electrode was placed on the forehead. CMAP amplitude was the peak to peak amplitude. Calculation of the degeneration ratio was as follows: $100 \times(1-$ amplitude on affected side / amplitude on unaffected side) [4]. The blink reflex test was conducted with the same EMG equipment under the following conditions: a pulse duration of $100 \mu \mathrm{s}$, a repetition rate of $1 \mathrm{~Hz}$, a bandwidth of $3 \mathrm{~Hz}$ to $10 \mathrm{kHz}$, a sensitivity of $500 \mu \mathrm{V} /$ division, and a sweep speed of 10 $\mathrm{ms} /$ division. Active electrodes were placed on the lower lid (orbicularis oculi muscles) with the patient in a supine position, a reference electrode was placed on the side of the nasal bones, and a ground electrode was placed in the middle of the chin. Stimuli were given to the supraor-

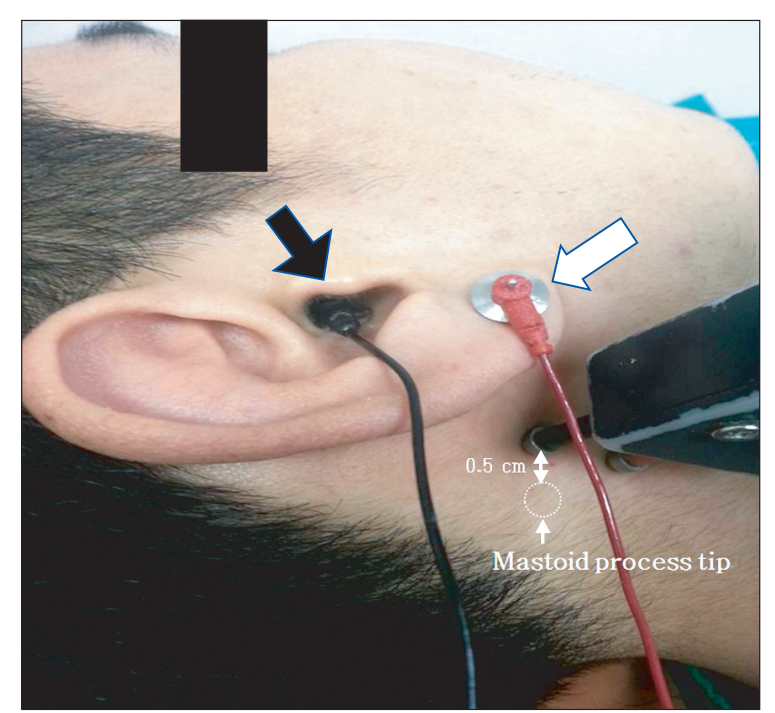

Fig. 1. Locations of active electrode (black arrow), reference electrode (white arrow), and stimulator. 

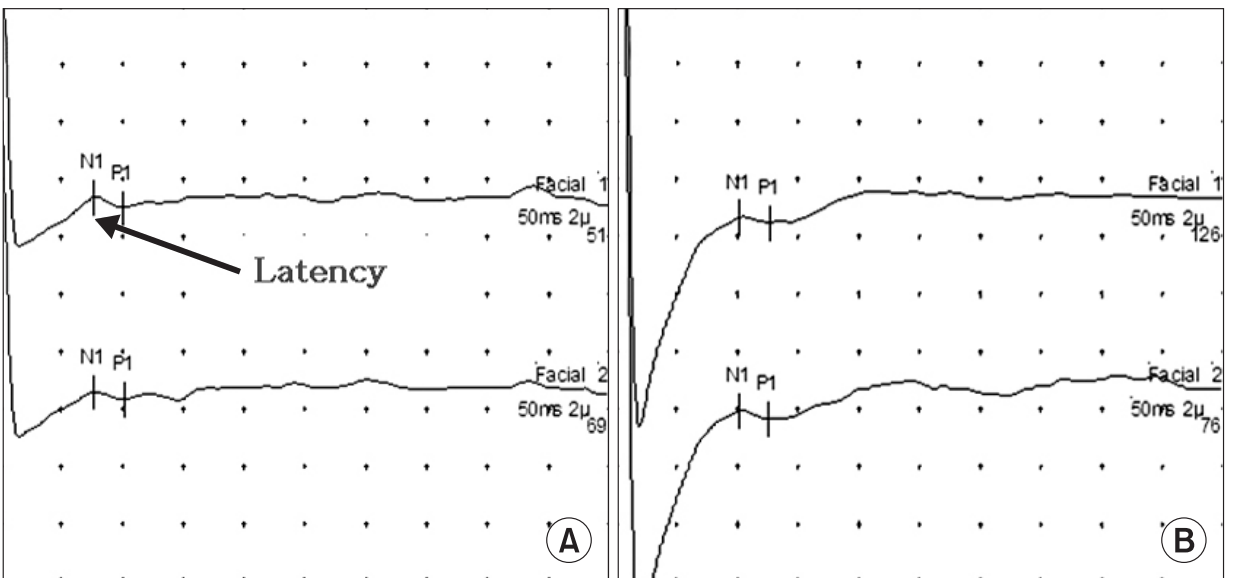

Fig. 2. Waveform of facial nerve antidromic evoked potential. (A) shows intact side and (B) shows affected side. bital notches using a stimulator at 20-40 mA.

For the FNAEP, we used same equipment as described above. The reference, active, and ground electrodes were placed on the earlobes, the posterior walls of the external auditory meatuses, and the dorsum of the hand respectively. Stimuli were given 50 times in total at a stimulus intensity of $20 \mathrm{mV}$ and a stimulus rate of $1 \mathrm{time} / \mathrm{s}$ at the stylomastoid foramen process [17,18] (Fig. 1). Sensitivity was adjusted to $2 \mu \mathrm{V} /$ division and data were recorded by an averaging technique. The signal was initially filtered at $30 \mathrm{~Hz}$ to $3 \mathrm{kHz}$ instead of $2 \mathrm{~Hz}$ to $10 \mathrm{kHz}$ as proposed in the preliminary study of Zhang et al. [17] to reduce noise and fluctuation of the base line. The results were recorded for both sides (Fig. 2).

In the control group, consisting of 19 normal adults, bilateral FNAEP latencies were measured in the same way, and the mean value of latency difference between bilateral sides was set as the reference value. The Wilcoxon signed-rank test was used to verify the significances of mean FNAEP latency differences between both sides in patients and the control group. Additionally, a check was kept on whether abnormal findings were observed in the patient group in the FNAEP and ENoG study. The McNemar test was used to ascertain whether the two methods differed significantly in diagnostic sensitivity. With the ANOVA test, an analysis was made of the correlation between ENoG and the House-Brackmann scale and between FNAEP latency differences and the HouseBrackmann scale.

All data were analyzed using the SPSS ver. 18.0 (SPSS Inc., Chicago, IL, USA). The significance level was set at $\mathrm{p}<0.05$.
Table 1. Demographic and clinical characteristics of subjects

\begin{tabular}{lcc}
\hline & $\begin{array}{c}\text { Patient } \\
\text { group }\end{array}$ & $\begin{array}{c}\text { Control } \\
\text { group }\end{array}$ \\
\hline Gender (male:female) & $10: 10$ & $15: 4$ \\
\hline Lesion side (right:left) & $12: 8$ & - \\
\hline Age (yr) & $35 \pm 18$ & $31 \pm 5$ \\
Disease duration (day) & $7.3 \pm 8.1$ & - \\
$\begin{array}{l}\text { House-Brackmann grading } \\
\text { system }\end{array}$ & & \\
$\quad$ Grade V & 3 & - \\
$\quad$ Grade IV & 6 & - \\
$\quad$ Grade III & 4 & - \\
$\quad$ Grade II & 7 & - \\
\hline
\end{tabular}

Values are presented as number or mean \pm standard deviation.

Table 2. Comparison of FNAEP latency between Bell's palsy patients and the controls

\begin{tabular}{lcc}
\hline & Patient group & Control group \\
\hline Latency difference $(\mathrm{ms})$ & $3.715(7.163)$ & $0.295(0.599)$ \\
p-value & $<0.01^{*}$ & 0.3 \\
\hline
\end{tabular}

Values are presented as number or mean (two standard deviation).

FNAEP, facial nerve antidromic evoked potential. ${ }^{*} \mathrm{p}<0.05$.

\section{RESULTS}

Patient characteristics and reference values

Demographic and clinical characteristics of subjects are presented in Table 1 . In the control group, the mean difference in FNAEP latency was $0.295 \mathrm{~ms}$ (2 standard 
deviation $[\mathrm{SD}]=0.599)$, which was used as the reference value in this study (Table 2). The mean of FNAEP latency was $10.392 \mathrm{~ms}(2 \mathrm{SD}=2.098)$. The mean age of the men $(\mathrm{n}=15)$ was 31.1 years $(2 \mathrm{SD}=6.2)$ and their latency averaged $10.603 \mathrm{~ms}(2 \mathrm{SD}=1.086)$. The mean age of the women $(n=4)$ was 29.5 years $(2 \mathrm{SD}=2.0)$ and their latency was $9.600 \mathrm{~ms}(2 \mathrm{SD}=4.082)$ on average. In all patients, FNAEP latency tended to be delayed on the affected side. Additionally, there were statistically significant differences between the affected and unaffected sides. In the control group, however, there was no significant difference in FNAEP latency between the sides (Table 2).

\section{FNAEP and ENoG sensitivity for Bell's palsy diagnosis}

In 8 of 20 patients who manifested clinical symptoms of Bell's palsy, the degeneration ratio was within the normal range $(<50 \%)$. Thus, it could not be ascertained whether they had definite facial nerve damage [10-12]. However, in those patients, the mean difference of FNAEP latency was $0.894 \mathrm{~ms}$ (reference value+2 SD) and above; thus it was judged to be abnormal, so it was possible to diagnose facial nerve damage. Diagnostic sensitivities were $90 \%$ and $60 \%$ for FNAEP and ENoG, respectively. Of the eight patients who were not demonstrated to have neurodegeneration by ENoG, six were examined within 7 days after the onset of symptoms, five were of House-Brackmann grade II, and three were of House-Brackmann grade IV, indicating that the FNAEP study may be more effective than ENoG at detecting facial nerve degeneration not only in the early stages but also in cases of mild symptoms. In contrast, in two patients whose FNAEP latencies were within the reference value, their facial nerve degeneration was confirmed because their degeneration ratios were above $50 \%$ (Table 3 ). However, with the McNemar test, there were no significant difference in diagnostic sensitivity between the tests $(\mathrm{p}=0.11)$.

Table 3. ENoG degeneration ratio and FNAEP latency difference in Bell's palsy patients

\begin{tabular}{ccc}
\hline \multirow{2}{*}{ FNAEP latency difference $(\mathrm{ms})$} & \multicolumn{2}{c}{$\begin{array}{c}\text { ENoG degeneration } \\
\text { ratio (\%) }\end{array}$} \\
\cline { 2 - 3 } & $\mathbf{2 5 0}$ & $<\mathbf{5 0}$ \\
\hline$>0.295 \pm 0.599$ & 10 & 8 \\
$\leq 0.295 \pm 0.599$ & 2 & 0 \\
\hline
\end{tabular}

ENoG, electroneurography; FNAEP, facial nerve antidromic evoked potential.
FNAEP and ENoG sensitivity for Bell's palsy diagnosis at early stage

In 10 patients who were examined within 7 days after the onset of symptoms, six patients show degeneration ratios within the normal range $(<50 \%)$. However, in all six patients, there were abnormal findings in relation to the FNAEP latency difference between the sides (Table 4). Diagnostic sensitivities were $100 \%$ and $40 \%$ for FNAEP and ENoG, respectively, on the McNemar test ( $\mathrm{p}=0.03$ ). These results showed that FNAEP was more statistically significantly effective than ENoG in detecting facial nerve damage within 7 days. In cases where abnormalities were found with both ENoG and FNAEP, the sensitivity was $50.0 \%$ in the patients. For the patients tested within 7 days, the sensitivity was $40.0 \%$.

\section{Blink reflex sensitivity for Bell's palsy diagnosis}

The blink reflex test was conducted in 19 of 20 patients: 18 showed abnormalities, and thus the sensitivity was $94.7 \%$. Of the nine patients who had been suffering from the disease for less than 7 days, eight showed abnormalities on the blink reflex test; thus the sensitivity was $88.9 \%$. The sensitivity was higher with the blink reflex test than with ENoG. In cases where ENoG and blink reflex test was performed altogether, the sensitivity was $52.6 \%$ in all patients. For the patients tested within 7 days, the sensitivity was $\mathbf{8 8 . 8 \%}$.

\section{FNAEP and ENoG for prognosis of Bell's palsy}

The House-Brackmann scale was applied to all 20 patients with Bell's palsy. The degeneration ratios averaged $32.5 \%, 59.8 \%, 62.8 \%$, and $78.6 \%$ in patients graded II, III, IV, and V, respectively. The degeneration ratio tended to increase as the House-Brackmann grade increased; this was statistically significant $(\mathrm{p}=0.006)$. However, there was no significant difference between House-Brackmann

Table 4. ENoG degeneration ratio and FNAEP latency difference in Bell's palsy patients within 7 days after onset

\begin{tabular}{ccc}
\hline \multirow{2}{*}{ FNAEP latency difference (ms) } & \multicolumn{2}{c}{$\begin{array}{c}\text { ENoG degeneration } \\
\text { ratio (\%) }\end{array}$} \\
\cline { 2 - 3 } & $\geq \mathbf{5 0}$ & $<\mathbf{5 0}$ \\
\hline$>0.295 \pm 0.599$ & 4 & 6 \\
$\leq 0.295 \pm 0.599$ & 0 & 0 \\
\hline
\end{tabular}

ENoG, electroneurography; FNAEP, facial nerve antidromic evoked potential. 
Table 5. Prognosis of Bell's palsy patients in one month

\begin{tabular}{|c|c|c|c|c|c|c|}
\hline \multirow[t]{2}{*}{ Subject no. } & \multirow[t]{2}{*}{ Gender } & \multirow[t]{2}{*}{ Age } & \multirow{2}{*}{$\begin{array}{l}\text { FNAEP latency difference } \\
\text { (ms) }\end{array}$} & \multirow{2}{*}{$\begin{array}{c}\text { ENoG } \\
\text { degeneration } \\
\text { ratio }(\%)\end{array}$} & \multicolumn{2}{|c|}{$\begin{array}{c}\text { House-Brackmann grading } \\
\text { system (grade) }\end{array}$} \\
\hline & & & & & Onset & $1 \mathrm{mo}$ \\
\hline 1 & F & 21 & $>0.295 \pm 0.599$ & $\geq 50$ & $\mathrm{~V}$ & II \\
\hline 2 & M & 36 & $>0.295 \pm 0.599$ & $\geq 50$ & III & II \\
\hline 3 & M & 25 & $>0.295 \pm 0.599$ & $\geq 50$ & IV & IV \\
\hline 4 & M & 47 & $>0.295 \pm 0.599$ & $<50$ & II & I \\
\hline 5 & $\mathrm{~F}$ & 41 & $>0.295 \pm 0.599$ & $<50$ & IV & I \\
\hline 6 & $\mathrm{~F}$ & 47 & $>0.295 \pm 0.599$ & $<50$ & II & I \\
\hline 7 & $\mathrm{~F}$ & 23 & $>0.295 \pm 0.599$ & $<50$ & IV & I \\
\hline 8 & M & 41 & $>0.295 \pm 0.599$ & $<50$ & II & I \\
\hline 9 & M & 39 & $\leq 0.295 \pm 0.599$ & $\geq 50$ & IV & IV \\
\hline 10 & $\mathrm{~F}$ & 28 & $\leq 0.295 \pm 0.599$ & $\geq 50$ & III & III \\
\hline
\end{tabular}

ENoG, electroneurography; FNAEP, facial nerve antidromic evoked potential.

grade and FNAEP latency difference, and FNAEP did not reflect the degree of facial nerve injury $(\mathrm{p}=0.181)$.

In 10 of the 20 patients, we checked whether symptoms had improved in a month. In the two patients whose FNAEP latencies were within the normal range, their symptoms did not improve on the House-Brackmann grading system, whereas in the five patients with normal ENoG findings, their symptoms improved a month later (Table 5). As a result, ENoG tended to be more closely associated with disease prognosis.

\section{DISCUSSION}

Unlike other electrodiagnostic tests, the FNAEP study makes it possible to check for facial nerve damage because it stimulates the outer part of the temporal bone and receives a signal from the inner part. FNAEP has the advantage of detecting facial nerve damage before it spreads to the outer part of the temporal bone.

In a previous study [13], 109 patients who underwent FNAEP within 7 days after Bell's palsy symptoms did not show triphasic waveforms, but showed abnormal waveforms. Of 88 patients who showed biphasic waveforms, 82 recovered fully. Nearly half of patients who showed monophasic waveforms or no response did not recover sufficiently. Thus, they suggested that FNAEP was highly effective in diagnosing the early stages of Bell's palsy and predicting the prognosis. However, their study focused on the morphology of the FNAEP waveforms, and therefore is directly comparable with this study focused on dif- ferences of latency between the sides.

Another study measured evoked potentials from the posterior walls of the external auditory meatuses and confirmed specific waveforms and prolonged latencies in facial palsy [14]. However, it is also not reasonable to compare their study conducted on animals with this study. Another study reported that three healthy people showed triphasic waves on FNAEP study, but that five of seven Bell's palsy patients revealed no response on the FNAEP study [15]. That study was conducted in a smaller number of patients than the present study, and moreover, did not deal with latency.

A recent study conducted on 46 patients with unilateral Bell's palsy reported that FNAEP enabled the detection of early facial nerve damage in the intratemporal bone, and that the prognosis could be predicted through the range of decrease in waveform amplitude and the increase in onset latencies [17]. Those results were consistent with this study: the increase in FNAEP latency is useful to detect facial nerve damage at an early stage. Our study is significant in that a comparison was made between FNAEP and ENoG regarding diagnostic value. In this study, the latency difference exceeded the reference value in 18 of 20 patients diagnosed with Bell's palsy, showing that latency should be used as a diagnostic scale in addition to FNAEP waveforms.

In this study conducted on 20 patients with Bell's palsy, not all cases could be detected by ENoG, but the eight undetected cases did show abnormal findings in the FNAEP study. Thus, it appears that FNAEP is useful in 
diagnosing Bell's palsy. In the case of 10 patients who had been suffering from early stage Bell's palsy, in particular, FNAEP showed abnormal findings in all of them, whereas ENoG revealed abnormalities in only four of them. Regarding the diagnostic sensitivity for facial nerve degeneration, FNAEP and ENoG showed $100 \%$ and $40 \%$, respectively, the difference was statistically significant $(\mathrm{p}=0.031)$. These results are consistent with a previous report that FNAEP was effective in detecting facial nerve damage that had not spread to the outer part of the temporal bones, and further supports present theories [17].

Regarding prognostic predictions, the degeneration ratio was superior to FNAEP latency. Patients who underwent ENoGs and whose degeneration ratios were within the normal range, showed good prognosis a month later. Patients whose FNAEP latency differences were within the reference value showed worse prognosis than those whose FNAEP latency differences were outside the reference value. In this study, unlike a previous study [17], FNAEP latency was found to be inappropriate for prognostic predictions.

This study has several limitations. Caution is needed in generalizing the results, given that this study was conducted on small numbers of patients and a control group. Second, this study did not thoroughly analyze the prognostic predictability of FNAEP latency because the follow-up was limited to 1 month after the onset of symptoms. Accordingly, a long-term follow-up and a large number of patients are needed to confirm FNAEP latency's prognostic value. Third, the FNAEP-recording method, used in this study, was proposed from a preliminary study by Zhang et al. [17]. It can be used with outpatients, without local anesthesia or needle electrode, but it differs from the more physiological method proposed by Nakatani et al. [13] and Kitani [15]. Thus, further studies are needed to compare them. Fourth, the short distance between the stimulation site and the recording site leads to the possibility of coactivating muscles around the recording site, causing contamination. In this regard, there is the need to reduce the activation of muscles around recording site, possibly with the use of muscle relaxants.

In conclusion, there were significant differences between ENoG and FNAEP study in diagnostic sensitivity for early stage Bell's palsy. It is important that FNAEP is monitored in patients with facial palsy at the early stage and integrated with other relevant test, such as ENoG.

\section{CONFLICT OF INTEREST}

No potential conflict of interest relevant to this article was reported.

\section{REFERENCES}

1. de Araujo MR, Azenha MR, Capelari MM, Marzola C. Management of Bell's palsy: a report of 2 cases. J Can Dent Assoc 2008;74:823-7.

2. Sittel C, Stennert E. Prognostic value of electromyography in acute peripheral facial nerve palsy. Otol Neurotol 2001;22:100-4.

3. Celik M, Forta H. Electrophysiological investigations and prognosis in idiopathic facial palsy. Electromyogr Clin Neurophysiol 1997;37:311-5.

4. Grosheva M, Wittekindt C, Guntinas-Lichius O. Prognostic value of electroneurography and electromyography in facial palsy. Laryngoscope 2008;118:394-7.

5. Grosheva M, Guntinas-Lichius O. Significance of electromyography to predict and evaluate facial function outcome after acute peripheral facial palsy. Eur Arch Otorhinolaryngol 2007;264:1491-5.

6. Chow LC, Tam RC, Li MF. Use of electroneurography as a prognostic indicator of Bell's palsy in Chinese patients. Otol Neurotol 2002;23:598-601.

7. Teudt IU, Nevel AE, Izzo AD, Walsh JT Jr, Richter CP. Optical stimulation of the facial nerve: a new monitoring technique? Laryngoscope 2007;117:1641-7.

8. Haginomori S, Wada S, Takamaki A, Nonaka R, Takenaka H, Takubo T. A new method for measuring compound muscle action potentials in facial palsy: a preliminary study. Muscle Nerve 2008;37:764-9.

9. Guo L, Jasiukaitis P, Pitts LH, Cheung SW. Optimal placement of recording electrodes for quantifying facial nerve compound muscle action potential. Otol Neurotol 2008;29:710-3.

10. Dumitru D, Amato AA, Zwarts M. Electrodiagnostic medicine. 2nd ed. Philadelphia: Hanley \& Belfus; 2002. Ch. 15, Electrodiagnostic medicine pitfalls; p. 541-77.

11. May M, Klein SR, Blumenthal F. Evoked electromyography and idiopathic facial paralysis. Otolaryngol Head Neck Surg 1983;91:678-85.

12. Aimoni C, Lombardi L, Gastaldo E, Stacchini M, Pastore A. Preoperative and postoperative electroneu- 
rographic facial nerve monitoring in patients with parotid tumors. Arch Otolaryngol Head Neck Surg 2003;129:940-3.

13. Nakatani H, Iwai M, Takeda T, Hamada M, Kakigi A, Nakahira M. Waveform changes in antidromic facial nerve responses in patients with Bell's palsy. Ann Otol Rhinol Laryngol 2002;111:128-34.

14. Tashima K, Takeda T, Nakatani H, Hamada M, Kakigi A. Antidromically evoked facial nerve response in guinea pigs: a long-term follow-up after nerve injury. ORL J Otorhinolaryngol Relat Spec 2010;71 Suppl 1:85-90.

15. Kitani S. Experimental study on antidromic evoked potential of the facial nerve for clinical application. Nihon Jibiinkoka Gakkai Kaiho 1994;97:645-53.

16. Dumitru D, Amato AA, Zwarts M. Electrodiagnostic medicine. 2nd ed. Philadelphia: Hanley \& Belfus; 2002. Ch. 17, Focal cranial neuropathies; p. 653-712.

17. Zhang WH, Chen MJ, Yang C, Zhang WJ. Prognostic value of facial nerve antidromic evoked potentials in bell palsy: a preliminary study. Int J Otolaryngol 2012;2012:960469.

18. Zhang WH, Chen MJ, Zhang WJ. Antidromica evoked potentials for positioning in facial paralysis. J Oral Sci Res 2011;8:676-9. 